Communications in Physics, Vol. 26, No. 2 (2016), pp. 99-109

DOI:10.15625/0868-3166/26/2/8639

\title{
SIMPLE EXPERIMENTAL SETUPS FOR OBSERVING WAVE-PARTICLE AND ENTANGLEMENT-SEPARABILITY SUPERPOSITIONS
}

\author{
NGUYEN BA AN ${ }^{\dagger}$ \\ Institute of Physics, Vietnam Academy of Science and Technology (VAST), \\ 18 Hoang Quoc Viet, Hanoi, Vietnam \\ ${ }^{\dagger} E$-mail: nban@iop.vast.vn \\ Received 23 August 2016 \\ Accepted for publication 22 September 2016
}

\begin{abstract}
It has been long believed that wave and particle cannot be superposed. Recently, however, modern quantum techniques have allowed one to prepare photon wave-particle superpositions experimentally. In this work, we suggest simple setups, which resort to neither any extra quantum resources nor controlled-Hadamard gates, to observe such superpositions as well as entanglement-separability superpositions with tunable entanglement degree. The suggested setups can also serve as an interaction-free method to detect partially absorbing objects.
\end{abstract}

Keywords: wave-particle superposition, entanglement-separability superposition.

Classification numbers: 03.65.Bz, 42.50.Dv.

\section{INTRODUCTION}

The microworld is governed by quantum mechanics which has been built on a set of postulates and principles. One of the most important principles is the complementarity principle conceived by Niels Bohr [1]. According to that principle, quantum states inherently possess complementary features which cannot be exposed simultaneously in one and the same experimental setup. Wave-like and particle-like nature are two such complementary properties of micro-objects. For instance, to observe a photon as a particle a device sketched in Fig. 1a is employed. A photon injected into path $a$ will be registered by either photodetector $D_{c}$ or photodetector $D_{d}$ with an equal probability of $1 / 2$, no mater what is the value of the phase shift $\varphi$. A click by $D_{c}\left(D_{d}\right)$ reveals the path $a \rightarrow c(b \rightarrow d)$ gone by the photon which must have been a particle. If a second balanced beam-splitter is placed as shown in Fig. $1 \mathrm{~b}$, then a click by $D_{c}$ or by $D_{d}$ may be equally triggered by the photon traveling along path $a \rightarrow c$ or path $b \rightarrow d$. That is, the photon's which-path information is erased by the second balanced beam-splitter and the probability that $D_{c}\left(D_{d}\right)$ clicks depends explicitly on $\varphi$, revealing the photon as a wave.

(C)2016 Vietnam Academy of Science and Technology 
Mystery is the fact that the photon's behavior turns out qualitatively different depending on which experimental setup (as in Fig. 1a or in Fig. 1b) is to be used to detect it! Albert Einstein disliked this counterintuitive phenomenon. Together with others he assumed that there must exist a kind of variable objectively hidden in the photon state that allows the photon before hitting the first balanced beam-splitter to "sense" the presence or absence of a second balanced beam-splitter in order to "decide" its behavior correspondingly. Later John Archibald Wheeler proposed gedanken experiments [2] in which random placement of the second balanced beamsplitter is delayed until after the photon had already passed through the first balanced beam-splitter. Several real experiments were successfully carried out in Wheeler's spirit (see, e.g., [3-5] and references therein), all of which supported the wave-particle complementarity. The hidden variable interpretation of Albert Einstein was thus ruled out by Wheeler's delayed-choice experiments.

A photon cannot show up as wave-like and particle-like at the same time because the setups in Fig. 1a and Fig. 1b exclude each other. This conclusion is, nevertheless, subjected to suspection when looking at the setups under the angle of quantum gates. Since a balanced beam-splitter acts like a Hadamard gate, Fig. 1a can operationally be replaced by Fig. 1c and Fig. 1b by Fig. 1d. Differently from Fig. 1c, Fig. 1d contains an additional Hadamard gate before the measurement process at the end. If one prepares an ancillary photon in a superposed state $\alpha|0\rangle+\beta|1\rangle$ and executes a controlled-Hadamard gate, followed by measuring both the photons (as seen in Fig. 1e), then wave and particle behavior can coexist simultaneously [6]. This original theoretical idea was realized in a number of complicated experiments using various physical means [7-12]. However, in the above-cited experiments the quantum resource cost increases since an extra photon is needed and, more seriously, a controlled-Hadamard gate is required. The latter requirement is severe because this is a two-photon gate whose implementation is technically challenging.

In this paper, we suggest much simpler setups to simultaneously observe photonic wavelike and particle-like nature. Our setups use only conventional optical devices readily available in laboratories and neither ancillary photons nor controlled-Hadamard gates are required. The same setups can also prepare entanglement-separability superpositions whose degree of entanglement is

adjustable by a variable parameter. In addition, our setups can serve as well as a method to detect not only fully but also partially absorbing objects without in any way interacting with them. In Section II we demonstrate how single- and two-photon wave-particle superpositions are generated by our setups, while Section III addresses the same issue for two-photon entanglement-separability superpositions. Conclusion is the final section.

\section{WAVE-PARTICLE SUPERPOSITIONS}

Our optical setups is schematically sketched in Fig. 1g. There are three inputs along paths $a, b, e$ and three outputs along paths $c, d, e$ which will be recognized by photodetector $D_{c}, D_{d}$, $D_{e}$, respectively. Let us inject a single photon into path $a$ but nothing into paths $b$ and $e$. That is, the input state has the form

$$
\left|\psi_{\text {in }}\right\rangle=|100\rangle_{\text {abe }} \text {. }
$$

First consider the case when $X$ is nothing. After passing the first balanced beam-splitter state $\left|\psi_{\text {in }}\right\rangle$ evolves to

$$
\left|\psi_{1}\right\rangle=\frac{1}{\sqrt{2}}\left(|10\rangle_{a b}+i|01\rangle_{a b}\right)|0\rangle_{e}
$$



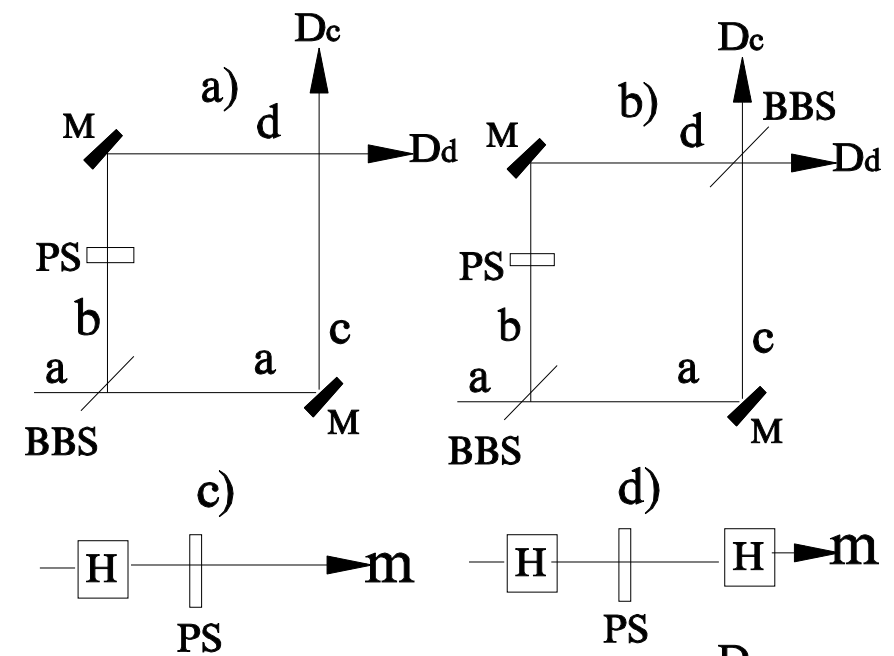

BBS d)

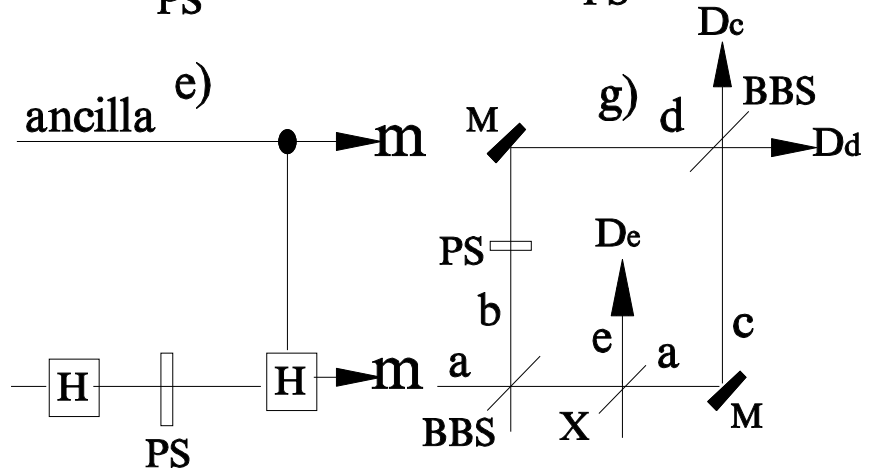

Fig. 1. Schematic for the experimental setups. BBS denotes a balanced beam-splitter, PS a phase-shifter, $\mathrm{M}$ a mirror, $D_{c(d, e)}$ a photodetector, $\mathrm{H}$ a Hadamard gate, $\mathrm{m}$ a measurement and $\mathrm{X}$ either nothing or a mirror or a general beam-splitter.

Since $P S|n\rangle_{b}=e^{i n \varphi}|n\rangle_{b}$ and $M|n\rangle_{b(a)}=i^{n}|n\rangle_{d(c)}$, after the phase-shifter and the mirrors state $\left|\psi_{1}\right\rangle$ is transformed to

$$
\left|\psi_{2}\right\rangle=\frac{1}{\sqrt{2}}\left(i|10\rangle_{c d}-e^{i \varphi}|01\rangle_{c d}\right)|0\rangle_{e}
$$

Then, after the second balanced beam-splitter, $\left|\psi_{2}\right\rangle \rightarrow\left|\psi_{\text {out }}^{X=n o t h i n g}\right\rangle$ with

$$
\left|\psi_{\text {out }}^{X=\text { nothing }}\right\rangle=e^{i \varphi / 2}\left[\sin (\varphi / 2)|10\rangle_{c d}-\cos (\varphi / 2)|01\rangle_{c d}\right]|0\rangle_{e}
$$


The probabilities $P_{c}^{X=n o t h i n g}, P_{d}^{X=n o t h i n g}$ and $P_{e}^{X=n o t h i n g}$ that the photodetectors $D_{c}, D_{d}$ and $D_{e}$ click are straightforwardly obtained from Eq. (4) as

$$
\begin{aligned}
& P_{c}^{X=\text { nothing }}=\sin ^{2}(\varphi / 2), \\
& P_{d}^{X=\text { nothing }}=\cos ^{2}(\varphi / 2), \\
& P_{e}^{X=\text { nothing }}=0,
\end{aligned}
$$

respectively. The explicit dependence of $P_{c}^{X=n o t h i n g}$ and $P_{d}^{X=n o t h i n g}$ on $\varphi$ is brought in by the quantum interference of the photon with itself at the second beam-splitter, i.e. the photon's which-path information (whether it traveled along path $c$ or path $d$ before being registered by a photodetector) is not known. Most pronounced is the case when $\varphi=0$ for which the outgoing photon always hits the photodetector $D_{d}$ and never hits $D_{c}$. This indicates the wave nature of the photon and we write $\left|\psi_{\text {out }}^{X=\text { nothing }}\right\rangle$ as

$$
\left|\psi_{\text {out }}^{X=\text { nothing }}\right\rangle=\left|\psi_{\text {wave }}\right\rangle_{c d}|0\rangle_{e}
$$

where

$$
\left|\psi_{\text {wave }}\right\rangle_{c d}=e^{i \varphi / 2}\left[\sin (\varphi / 2)|10\rangle_{c d}-\cos (\varphi / 2)|01\rangle_{c d}\right]
$$

specifying the photon as a wave.

Next, consider the case when $X$ is a mirror. Then, instead of $\left|\psi_{\text {out }}^{X=n o t h i n g}\right\rangle$ we have

$$
\left|\psi_{\text {out }}^{X=\text { mirror }}\right\rangle=-\frac{1}{2} e^{i \varphi}\left(i|10\rangle_{c d}+|01\rangle_{c d}\right)|0\rangle_{e}+\frac{i}{\sqrt{2}}|00\rangle_{c d}|1\rangle_{e}
$$

from which it immediately follows

$$
\begin{aligned}
& P_{c}^{X=\text { mirror }}=P_{d}^{X=\text { mirror }}=\frac{1}{4}, \\
& P_{e}^{X=\text { mirror }}=\frac{1}{2} .
\end{aligned}
$$

Since all the above detection probabilities are independent of $\varphi$, the photon in this situation behaves like a particle: no quantum interference occurs at the second beam-splitter because the photon's which-path information is always disclosed. Namely, if $D_{e}$ clicks then the photon must have traveled along path $a$ and if $D_{e}$ does not click then it must have traveled along path $b$ (i.e., in any case it is sure that the photon traveled only along one of the paths as a particle would do). It is interesting to notice that when $\varphi=0$ we have $P_{c}^{X=n o t h i n g}=0\left(P_{d}^{X=n o t h i n g}=1\right)$. The fact of $P_{c}^{X=\text { mirror }}=1 / 4>0$ even when $\varphi=0$ signals the presence of a mirror (or any absolutely absorbing object) in path $a$, although the photon had not gone at all along that path. Such a phenomenon was known in the literature as the interaction-free measurement that provides a method to "detect" a dangerous absorbing object like a sensitive bomb without "touching" it [13]. The above argumentation allows us to introduce the photon particle state

$$
\left|\psi_{\text {particle }}\right\rangle_{c d}=-\frac{1}{\sqrt{2}} e^{i \varphi}\left(i|10\rangle_{c d}+|01\rangle_{c d}\right)
$$


in terms of which $\left|\psi_{\text {out }}^{X=\text { mirror }}\right\rangle$ is rewritten in the form

$$
\left|\psi_{\text {out }}^{X=\text { mirror }}\right\rangle=\frac{1}{\sqrt{2}}\left(\left|\psi_{\text {particle }}\right\rangle_{c d}|0\rangle_{e}+i|00\rangle_{c d}|1\rangle_{e}\right) \text {. }
$$

The above analysis clearly shows that, depending on the detection setup, the photon may behave either as a particle (if a mirror is inserted) or a wave (if the mirror is removed). To observe both wave-like and particle-like behaviors of the photon at the same time by a single experimental setup we insert in path $a$ a general beam-splitter, i.e., $X=G B S$. A general beam-splitter has transmittance $t$, with $0 \leq t \leq 1$. All the following formulae aim at the case with $0<t<1$. They are however applicable to any $t \in[0,1]$, thus covering the cases when $X$ is nothing $(t=1)$ and when $X$ is a mirror $(t=0)$ as particular situations. The actions of a general beam-splitter on a photon state $\operatorname{read}|01\rangle_{a b(c d)} \rightarrow t|01\rangle_{a b(c d)}+i \sqrt{1-t^{2}}|10\rangle_{a b(c d)}$ and $|10\rangle_{a b(c d)} \rightarrow i \sqrt{1-t^{2}}|01\rangle_{a b(c d)}+t|10\rangle_{a b(c d)}$. By direct calculations we arrived at the following expression for the output state

$$
\left|\psi_{\text {out }}^{X=G B S}\right\rangle=\frac{1}{2}\left[i\left(t-e^{i \varphi}\right)|10\rangle_{c d}-\left(t+e^{i \varphi}\right)|01\rangle_{c d}\right]|0\rangle_{e}+\frac{i \sqrt{1-t^{2}}}{\sqrt{2}}|00\rangle_{c d}|1\rangle_{e}
$$

The corresponding detection probabilities are then

$$
\begin{aligned}
P_{c}^{X=G B S} & =\frac{1}{4}\left(1-2 t \cos (\varphi)+t^{2}\right), \\
P_{d}^{X=G B S} & =\frac{1}{4}\left(1+2 t \cos (\varphi)+t^{2}\right), \\
P_{e}^{X=G B S} & =\frac{1}{2}\left(1-t^{2}\right) .
\end{aligned}
$$

Making use of Eqs. (9) and (13) we can reexpress $\left|\psi_{\text {out }}^{X=G B S}\right\rangle$ in Eq. (15) as

$$
\left|\psi_{\text {out }}^{X=G B S}\right\rangle=\left(t\left|\psi_{\text {wave }}\right\rangle_{c d}+\frac{1-t}{\sqrt{2}}\left|\psi_{\text {particle }}\right\rangle_{c d}\right)|0\rangle_{e}+\frac{i \sqrt{1-t^{2}}}{\sqrt{2}}|00\rangle_{c d}|1\rangle_{e} .
$$

Hence, with a probability of $\left(1+t^{2}\right) / 2$ the photodetector $D_{e}$ is silent and $\left|\psi_{\text {out }}^{X=G B S}\right\rangle$ collapses into

$$
\left|\psi_{w-p}\right\rangle_{c d}=\frac{\sqrt{2}}{\sqrt{1+t^{2}}}\left(t\left|\psi_{\text {wave }}\right\rangle_{c d}+\frac{1-t}{\sqrt{2}}\left|\psi_{\text {particle }}\right\rangle_{c d}\right)
$$

which is transparently a wave-particle superposition state, i.e., both wave-like and particle-like properties are exhibited simultaneously. The photon wave-particle behavior is also reflected in the detection probabilities which can be seen by rewriting $P_{c(d)}^{X=G B S}$ in terms of $P_{c(d)}^{X=\text { nothing }}$ (see Eqs. (5) and (6)) and $P_{c(d)}^{X=\text { mirror }}($ see (11)):

$$
P_{c(d)}^{X=G B S}=t^{2} P_{c(d)}^{X=\text { nothing }}+(1-t)^{2} P_{c(d)}^{X=\text { mirror }}+4 t(1-t) P_{c(d)}^{X=\text { nothing }} P_{c(d)}^{X=\text { mirror }} .
$$

In the right-hand-side of Eq. (21) the first term is due to the contribution of the wave nature of the photon, the second term is due to the contribution of the particle nature, while the last term describes their quantum interference which occurs for $0<t<1$. The dependences of $P_{c(d)}^{X=G B S}$ on $t$ and $\varphi$ are displayed in Fig. 2, showing clearly the particle-like (wave-like) behavior at $t=0$ $(t=1)$ and the continuous morphing from the particle-like (wave-like) to the wave-like (particlelike) behavior as $t$ gradually varies from $0(1)$ to $1(0)$. The special result that $P_{c}^{X=G B S}>0$ when 
$0<t<1$ even for $\varphi=0$ is significant. Transparently, it gives rise to a possibility of "detecting" a partially absorptive object (i.e., an object which partially transmits and partially absorbs the input signal) without "touching" it. Conventionally, the method to detect a fully absorptive object (i.e., an object which $100 \%$ absorbs and never transmits any input signal) without the need of sending a signal through it was discovered in Ref. [13] and commonly referred to in the literature as the "interaction-free measurement" method. Therefore, the possibility of "detecting" a partially absorptive object without "touching" it (as described above) widdens the applicability of the wellknown "interaction-free measurement" method [13] in which the to-be-detected object must be fully absorptive.
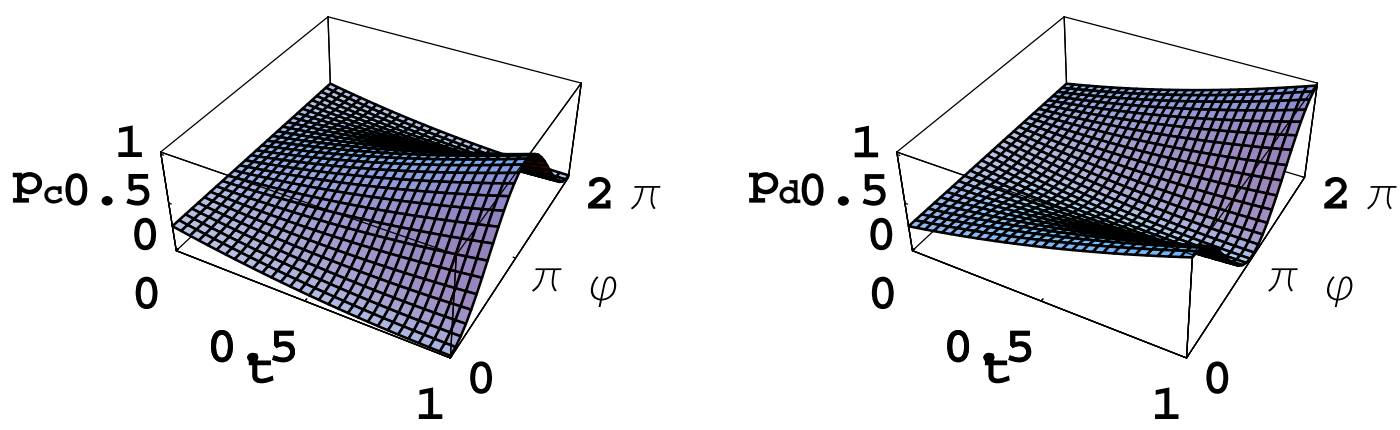

Fig. 2. $P_{c(d)}=P_{c(d)}^{X=G B S}$ as a function of $t$ and $\varphi$. For $t=0(t=1)$ the photon behaves as a particle (wave), but for $0<t<1$ it manifests itself as a particle and a wave at the same time. A continuous morphing between the two extreme behaviors happens when $t$ is being swept from 0 to 1 or vice versa.

We now turn to the case when two photons are injected to the device, one into path $a$ and the other into path $b$ leaving path $e$ empty. The input state is

$$
\left|\Psi_{\text {in }}\right\rangle=|11\rangle_{a b}|0\rangle_{e} .
$$

If $X$ is nothing the output state can be derived as

$$
\left|\Psi_{\text {out }}^{X=\text { nothing }}\right\rangle=\left|\Psi_{\text {wave }}\right\rangle_{c d}|0\rangle_{e}
$$

where

$$
\left|\Psi_{\text {wave }}\right\rangle_{c d}=\frac{1}{\sqrt{2}} e^{i \varphi}\left[\sin \varphi\left(|02\rangle_{c d}-|20\rangle_{c d}\right)+\sqrt{2} \cos \varphi|11\rangle_{c d}\right]
$$

denotes the two-photon wave state since the detection probabilities are $\varphi$-dependent. Namely, let $P_{k l m}^{X=n o t h i n g}$ be the probability that the detectors $D_{c}, D_{d}$ and $D_{e}$ register $k, l$ and $m$ photons, respectively, then

$$
\begin{gathered}
P_{020}^{X=\text { nothing }}=P_{200}^{X=\text { nothing }}=\frac{\sin ^{2} \varphi}{2}, \\
P_{110}^{X=n o t h i n g}=\cos ^{2} \varphi
\end{gathered}
$$


which exhibit interference patterns.
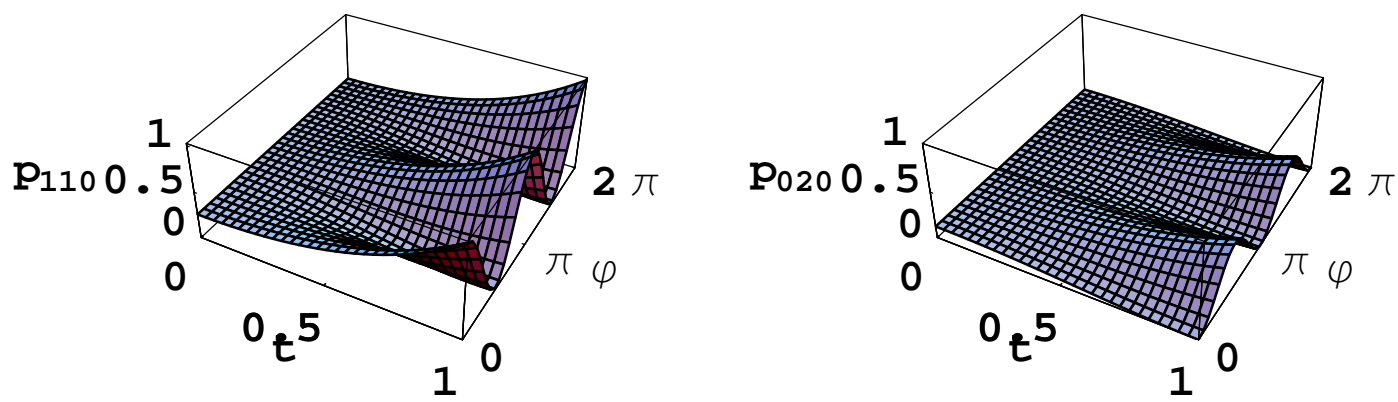

Fig. 3. $P_{110(020)}=P_{110(020)}^{X=G B S}$ as a function of $t$ and $\varphi$. For $t=0(t=1)$ the two photons behave as particles (waves), but for $0<t<1$ they show up as particle-like and wave-like at the same time. A continuous morphing between the two extreme behaviors happens when $t$ is being swept from 0 to 1 or vice versa.

If $X$ is a mirror the output state can be derived as

$$
\left|\Psi_{\text {out }}^{X=\text { mirror }}\right\rangle=-\frac{i}{\sqrt{2}}|00\rangle_{c d}|2\rangle_{e}-\frac{i}{2 \sqrt{2}} e^{2 i \varphi}\left(|02\rangle_{c d}+i \sqrt{2}|11\rangle_{c d}-|20\rangle_{c d}\right)|0\rangle_{e} .
$$

The concerned detection probabilities are

$$
\begin{gathered}
P_{020}^{X=\text { mirror }}=P_{200}^{X=\text { mirror }}=\frac{1}{8}, \\
P_{110}^{X=\text { mirror }}=\frac{1}{4}, \\
P_{002}^{X=\text { mirror }}=\frac{1}{2},
\end{gathered}
$$

which are independent of $\varphi$, exhibiting the particle-like behavior. In case $D_{e}$ does not click, which happens with a probability of $1 / 2$, the state before $D_{c}$ and $D_{d}$ collapses into

$$
\left|\Psi_{\text {particle }}\right\rangle_{c d}=\frac{1}{2} e^{2 i \varphi}\left(|02\rangle_{c d}+i \sqrt{2}|11\rangle_{c d}-|20\rangle_{c d}\right),
$$

and $\left|\Psi_{\text {out }}^{X=\text { mirror }}\right\rangle$ can be reformulated in the form

$$
\left|\Psi_{\text {out }}^{X=\text { mirror }}\right\rangle=-\frac{i}{\sqrt{2}}\left(\left|\Psi_{\text {particle }}\right\rangle_{c d}|0\rangle_{e}+|00\rangle_{c d}|2\rangle_{e}\right) .
$$


If $X$ is a general beam splitter the output state can be derived as

$$
\begin{aligned}
\left|\Psi_{\text {out }}^{X=G B S}\right\rangle= & \frac{1}{2}\left(t^{2}+e^{2 i \varphi}\right)|11\rangle_{c d}|0\rangle_{e}+\frac{i}{2 \sqrt{2}}\left(t^{2}-e^{2 i \varphi}\right)\left(|02\rangle_{c d}-|20\rangle_{c d}\right)|0\rangle_{e} \\
& +t \sqrt{\frac{1-t^{2}}{2}}\left(|01\rangle_{c d}-i|10\rangle_{c d}\right)|1\rangle_{e}-\frac{i}{\sqrt{2}}\left(1-t^{2}\right)|00\rangle_{c d}|2\rangle_{e},
\end{aligned}
$$

leading to

$$
\begin{gathered}
P_{020}^{X=G B S}=P_{200}^{X=G B S}=\frac{1}{8}\left[1-2 t^{2} \cos (2 \varphi)+t^{4}\right], \\
P_{110}^{X=G B S}=\frac{1}{4}\left[1+2 t^{2} \cos (2 \varphi)+t^{4}\right], \\
P_{011}^{X=G B S}=P_{101}^{X=G B S}=\frac{1}{2} t^{2}\left(1-t^{2}\right), \\
P_{002}^{X=G B S}=\frac{1}{2}\left(1-t^{2}\right)^{2} .
\end{gathered}
$$

In case $D_{e}$ does not click, which happens with a probability of $\left(1+t^{4}\right) / 2$, the state $\left|\Psi_{\text {out }}^{X=G B S}\right\rangle$ collapses into

$$
\begin{aligned}
\left|\Psi_{w-p}\right\rangle_{c d}= & \frac{1}{2} \sqrt{\frac{1}{1+t^{4}}}\left[\sqrt{2}\left(t^{2}+e^{2 i \varphi}\right)|11\rangle_{c d}\right. \\
& +i\left(t^{2}-e^{2 i \varphi}\right)\left(|02\rangle_{c d}-|20\rangle_{c d}\right]
\end{aligned}
$$

and $\left|\Psi_{\text {out }}^{X=G B S}\right\rangle$ can be represented as

$$
\begin{aligned}
\left|\Psi_{\text {out }}^{X=G B S}\right\rangle= & \frac{\sqrt{1+t^{4}}}{\sqrt{2}}\left|\Psi_{w-p}\right\rangle_{c d}|0\rangle_{e} \\
& +t \sqrt{\frac{1-t^{2}}{2}}\left(|01\rangle_{c d}-i|10\rangle_{c d}\right)|1\rangle_{e} \\
& -\frac{i}{\sqrt{2}}\left(1-t^{2}\right)|00\rangle_{c d}|2\rangle_{e} .
\end{aligned}
$$

The fact that the state (38) is a two-photon wave-particle superposition can be recognized by using (24), (31) to decompose (38) as

$$
\left|\Psi_{w-p}\right\rangle_{c d}=\sqrt{\frac{1}{1+t^{4}}}\left(t^{2} \sqrt{2}\left|\Psi_{\text {wave }}\right\rangle_{c d}-i\left(1-t^{2}\right)\left|\Psi_{\text {particle }}\right\rangle_{c d}\right)
$$

as well as by using (25), (26) and (28), (29) to decompose (34), (35) as

$$
\begin{aligned}
P_{020(200,110)}^{X=G B S}= & t^{4} P_{020(200,110)}^{X=\text { nothing }}+\left(1-t^{2}\right)^{2} P_{020(200,110)}^{X=\text { mirror }} \\
& +4 t^{2}\left(1-t^{2}\right) P_{020(200,110)}^{X=\text { nothing }} P_{020(200,110)}^{X=\text { mirror }} .
\end{aligned}
$$

The probabilities $P_{020(200,110)}^{X=G B S}$ as functions of $t$ and $\varphi$ are plotted in Fig. 3. 


\section{ENTANGLEMENT-SEPARABILITY SUPERPOSITIONS}

A multipartite quantum state may be either entangled or separable depending on the specific setup which is used to prepare it. As was long thought, the setup that prepares an entangled state and the setup that prepares a separable state mutually exclude each other and cannot be performed simultaneously. Then, in every preparation run the quantum state definitely appears either as entangled or as separable. In other words, the piece of information ("entanglement" or "separability") obtainable from such incompatible preparation processes are complementary, similar to "particle" or "wave" properties. In what follows, we use the same single setup as presented in the previous section with the same input state (22) to demonstrate that a tunable superposition of "entanglement" and "separability" can be realized. For that purpose we set $\varphi=0$ and are interested in the case when $D_{e}$ is silent. If so, the total output state $\left|\Psi_{\text {out }}^{X}\right\rangle$ collapses into $\left|\Psi_{\text {out }}^{X}(\varphi=0)\right\rangle_{c d}|0\rangle_{e}$. Then, for $X=$ nothing, $\left|\Psi_{\text {out }}^{X=\text { nothing }}(\varphi=0)\right\rangle_{c d}=\left|\Psi_{\text {separable }}\right\rangle_{c d}$, with

$$
\left|\Psi_{\text {separable }}\right\rangle_{c d}=|11\rangle_{c d}
$$

being a separable state. As for $X=$ mirror, we get $\left|\Psi_{\text {out }}^{X=\text { mirror }}(\varphi=0)\right\rangle_{c d}=\left|\Psi_{\text {entangled }}\right\rangle_{c d}$, with

$$
\left|\Psi_{\text {entangled }}\right\rangle_{c d}=\frac{1}{2}(|02\rangle+i \sqrt{2}|11\rangle-|20\rangle)_{c d}
$$

being a bipartite entangled state. It is seen that the final two-photon state will with certainty be either separable or entangled conditioned on the specific preparation setup ( $X=$ nothing or $X=$ mirror).

To realize a superposition of $\left|\Psi_{\text {separable }}\right\rangle_{c d}$ and $\left|\Psi_{\text {entangled }}\right\rangle_{c d}$ by a single setup, we set $X=G B S$, in which case we obtain $\left|\Psi_{o u t}^{X=G B S}(\varphi=0)\right\rangle_{c d}=\left|\Psi_{s-e}\right\rangle_{c d}$, with

$$
\left|\Psi_{s-e}\right\rangle_{c d}=\sqrt{\frac{1}{1+t^{4}}}\left(t^{2} \sqrt{2}\left|\Psi_{\text {separable }}\right\rangle_{c d}-i\left(1-t^{2}\right)\left|\Psi_{\text {entan } g l e d}\right\rangle_{c d}\right) .
$$

The state (44) is actually an entanglement-separability superposition. Note that the entanglement amount contained in state $\left|\Psi_{\text {entangled }}\right\rangle_{c d}$ is fixed, whereas the entanglement amount contained in state $\left|\Psi_{s-e}\right\rangle_{c d}$ is tunable by varying the value of $t$. To make it clearer let us assess the entanglement amount using the so-called entropy of entanglement, which for an arbitrary bipartite state $|\Phi\rangle_{c d}$ is defined by

$$
E o E\left(|\Phi\rangle_{c d}\right)=-\sum_{j=0}^{2} \lambda_{j} \log _{2}\left(\lambda_{j}\right),
$$

with $\lambda_{j}$ being the eigenvalues of the reduced density matrix $\rho=\operatorname{Tr}_{c}|\Phi\rangle_{c d}\left\langle\Phi\left|=\operatorname{Tr}_{d}\right| \Phi\right\rangle_{c d}\langle\Phi|$. For $|\Phi\rangle_{c d}=\left|\Psi_{s-e}\right\rangle_{c d}$, it is easy to calculate the concerned eigenvalues whose explicit expressions in terms of $t$ are

$$
\begin{aligned}
& \lambda_{0}=\frac{1}{4} \frac{\left(1-t^{2}\right)^{2}}{\left(1+t^{4}\right)}, \\
& \lambda_{1}=\frac{1}{2} \frac{\left(1+t^{2}\right)^{2}}{\left(1+t^{4}\right)},
\end{aligned}
$$

and

$$
\lambda_{2}=\lambda_{0}
$$




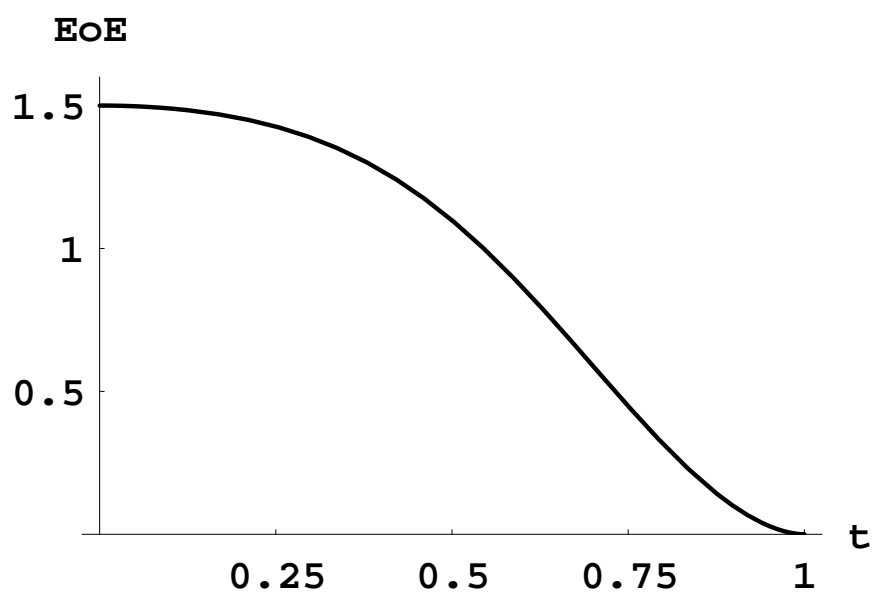

Fig. 4. Entropy of entanglement $E o E$ of $\left|\Psi_{s-e}\right\rangle_{c d}$, Eq. (45), versus $t$. The entanglement degree contained in $\left|\Psi_{s-e}\right\rangle_{c d}$ can be adjusted by choosing an appropriate value of $t$.

Figure 4 plots $E o E\left(\left|\Psi_{s-e}\right\rangle_{c d}\right)$ as a function of $t$. As it should be, for $t=0$ the value of $E o E\left(\left|\Psi_{s-e}\right\rangle_{c d}\right)$ coincides with $E o E\left(\left|\Psi_{\text {entangled }}\right\rangle_{c d}\right)=1.5$, while for $t=1$ the value of $E o E\left(\left|\Psi_{s-e}\right\rangle_{c d}\right)$ coincides with $E o E\left(\left|\Psi_{\text {separable }}\right\rangle_{c d}\right)=0$. A desired amount of entanglement between 0 and 1.5 can be obtained by adjusting the value of $t$.

\section{CONCLUSION}

The long-standing complementarity principle needs be refined because two complementary properties such as wave and particle or entanglement and separability can manifest themselves at the same time in a single measuring configuration as confirmed by a number of famous modern experiments [7-12]. We studied these experiments and found that they all mandatorily required (i) ancillary qubits, (ii) controlled-Hadamard gates and (iii) measurement of the ancillas. To reduce the overall cost as well as to facilitate the performance, we proposed simpler experimental setups which are free from the three above-mentioned requirements. Ours are thus both economical and less challenging technically since no additional quantum resources are necessary and only standard optical elements such as beam-splitters, phase-shifters, mirrors and photodetectors are employed. It is hoped that the setups presented here could be of certain interest for experimentalists.

\section{ACKNOWLEDGMENTS}

This work is supported by Vietnam Foundation for Science and Technology Development (NAFOSTED) under Project No. 103.01-2014.02.

\section{REFERENCES}

[1] N. Bohr, Nature 121 (1928) 580.

[2] J. A. Wheeler, The 'Past' and the 'Delayed-Choice Double-Slit Experiment', p. 948, in A. R. Marlow, editor, Mathematical Foundations of Quantum Theory, Academic Press, 1978.

[3] V. Jacques, E. Wu, F. Grosshans, F. Treussart, P. Grangier, A. Aspect and J. F. Roch, Science 315 (2007) 966. 
[4] V. Jacques, E. Wu, F. Grosshans, F. Treussart, P. Grangier, A. Aspect and J. F. Roch, Phys. Rev. Lett. 100 (2008) 220402.

[5] X. S. Ma, J. Kofler and A. Zeilinger, Rev. Mod. Phys. 88 (2016) 015005.

[6] R. Ionicioiu and D. R. Terno, Phys. Rev. Lett. 107 (2011) 230406.

[7] S. S. Roy, A. Shukla and T. S. Mahesh, Phys. Rev. A 85 (2012) 022109.

[8] R. Auccaise, R. M. Serra, J. G. Filgueiras, R. S. Sarthour, I. S. Oliveira and L. C. Céler, Phys. Rev. A 85 (2012) 032121.

[9] G. Adesso and D. Girolami, Nature Photonics 6 (2012) 579.

[10] J. S. Tang, Y. L. Li, X. Y. Xu, G. Y. Xiang, C. F. Li and G. C. Guo, Nature Photonics 6 (2012) 600.

[11] A. Peruzzo, P. Shadbolt, N. Brunner, S. Popescu, J. L. OBrien, Science 338 (2012) 634.

[12] F. Kaiser, T. Coudreau, P. Milman, D. B. Ostrowsky, and S. Tanzilli, Science 338 (2012) 637.

[13] P. Kwiat, H. Weinfurter, T. Herzog and A. Zeilinger, Phys. Rev. Lett. 74 (1995) 4763. 
